PROCEEDINGS OF THE

AMERICAN MATHEMATICAL SOCIETY

Volume 130, Number 9 , Pages $2553-2558$

S 0002-9939(02)06364-5

Article electronically published on February 4, 2002

\title{
CHARACTER DEGREE SETS THAT DO NOT BOUND THE CLASS OF A $p$-GROUP
}

\author{
I. M. ISAACS AND M. C. SLATTERY
}

(Communicated by Stephen D. Smith)

\begin{abstract}
Suppose that we are given a set $\mathcal{S}$ of powers of a prime $p$ and that $1 \in \mathcal{S}$. A technique is presented that enables the construction of a $p$-group of specified nilpotence class $n$ such that its set of irreducible character degrees is exactly $\mathcal{S}$. If $|\mathcal{S}| \geq 2$, then this can be done for $2 \leq n \leq p$ and if $p \in \mathcal{S}$, then the only requirement is $2 \leq n$.
\end{abstract}

\section{IntRoduCtion}

If $G$ is a finite group, then as usual, we write $\operatorname{cd}(G)$ to denote the set of degrees of the irreducible characters of $G$. Now fix some prime $p$ and let $\mathcal{S}$ be a finite set of powers of $p$, subject only to the condition that $1 \in \mathcal{S}$. It is known that there necessarily exists some $p$-group $P$ such that $\operatorname{cd}(P)=\mathcal{S}$, and in fact, it is always possible to choose $P$ so that its nilpotence class $c(P)$ is at most 2. (This is the main result of [2].)

For some choices of the set $\mathcal{S}$, there exists an upper bound on the nilpotence classes of those $p$-groups $P$ for which $\operatorname{cd}(P)=\mathcal{S}$, while for other sets $\mathcal{S}$, there is no such bound. For example, it was shown in [1] that if $\operatorname{cd}(P)=\left\{1, p^{e}\right\}$, then the class of $P$ is at most $p$ if $e>1$, but that the class of $P$ can be unboundedly large if $e=1$. (The problem of determining which sets of powers of $p$ imply an upper bound on the nilpotence class of a $p$-group having that degree set was first suggested by the second author in his paper [6].)

We shall say that a set $\mathcal{S}$ of powers of $p$ (containing 1) is class bounding if there exists an upper bound (depending on $\mathcal{S}$, of course) for the nilpotence classes of all $p$-groups $P$ such that $\operatorname{cd}(P)=\mathcal{S}$. By the result mentioned in the previous paragraph, for example, we see that if $|\mathcal{S}|=2$, then $|\mathcal{S}|$ is class bounding if and only if $p \notin \mathcal{S}$. It was proved in [5] that the corresponding result also holds when $|\mathcal{S}|=3$. In this case too, the set $\mathcal{S}$ is class bounding if and only if $p \notin \mathcal{S}$. Other class-bounding sets were constructed in [5], including some having arbitrarily large cardinality, but all known class-bounding sets of powers of $p$ fail to contain the number $p$.

Of course, these results suggest the general conjecture that an arbitrary set $\mathcal{S}$ of powers of $p$ (containing 1 ) is class bounding if and only if $p \notin \mathcal{S}$. The main result of this paper establishes the "only if" part of this conjecture.

Received by the editors February 23, 2001 and, in revised form, April 16, 2001.

2000 Mathematics Subject Classification. Primary $20 \mathrm{C} 15$.

The research of the first author was partially supported by the U. S. National Security Agency. 
Theorem A. Let $p$ be any prime, let $n \geq 2$ be an integer and suppose that $\mathcal{S}$ is a finite set of powers of $p$ containing both 1 and $p$. Then there exists a p-group $P$ with nilpotence class $n$ and $\operatorname{cd}(P)=\mathcal{S}$.

Our proof of Theorem A uses a variation of the technique used in [5] to prove the corresponding result when $|\mathcal{S}|=3$.

\section{The CONSTRUCTION}

Our principal construction tool is the well known "pullback", as is described in the following lemma.

(2.1) Lemma. Let $A \triangleleft X$ and $B \triangleleft Y$ be groups such that $X / A \cong Y / B$. Then there exists a group $P$ having normal subgroups $R$ and $S$ with the following properties:

(a) There exist isomorphisms $\alpha: P / R \rightarrow X$ and $\beta: P / S \rightarrow Y$.

(b) $R \cap S=1$.

(c) $\alpha(R S / R)=A$ and $\beta(R S / S)=B$.

Proof. Write $\bar{X}=X / A$ and $\bar{Y}=Y / B$ and let $\theta: \bar{X} \rightarrow \bar{Y}$ be the given isomorphism. Let $\Gamma=X \times Y$ be the external direct product and take $P$ to be the subgroup of $\Gamma$ consisting of all pairs $(x, y)$ such that $\theta(\bar{x})=\bar{y}$. It is clear that for each element $x \in X$, there exists an element $y \in Y$ such that $(x, y)$ lies in $P$, and since $\theta$ is surjective, we see that the reverse is true too: For each element $y \in Y$, there exists $x \in X$ such that $(x, y) \in P$. In other words, the projection homomorphisms from $P$ to $X$ and from $P$ to $Y$ are surjective. We let $R$ and $S$ be respectively the kernels of these homomorphisms and we let $\alpha$ and $\beta$ be the corresponding isomorphisms from $P / R$ onto $X$ and from $P / S$ onto $Y$. We have now established (a).

The first component of an arbitrary element of $R$ is 1 , and hence the second component can be any element $y \in Y$ such that $\theta(\overline{1})=\bar{y}$. In other words, the second component of an element of $R$ is an arbitrary element of $B$, and thus $R=1 \times B$. Also, since $\theta$ is injective, we see that $S=A \times 1$, and in particular, it is clear that $R \cap S=1$, as required.

Now $\alpha(R S / R)$ is the image of $R S=A \times B$ under the projection to $X$, and it follows that $\alpha(R S / R)=A$. Similarly, $\beta(R S / S)=B$, and the proof is complete.

In the notation of Lemma 2.1, it is easy to see that if $X$ and $Y$ are $p$-groups, then $P$ is also a $p$-group. Furthermore, it follows from the facts that $P / R \cong X$, $P / S \cong Y$ and $R \cap S=1$ that the nilpotence class $c(P)=\max \{c(X), c(Y)\}$.

(2.2) Definition. Let $a \geq 0$ be an integer. We will say that a $p$-group $P$ is suitable with respect to $a$ if $P$ has an abelian normal subgroup $A$ such that the following hold:

(1) $P / A$ is elementary abelian of order $p^{a}$.

(2) Every linear character of $A$ extends to its stabilizer in $P$.

Note that if $P$ is suitable with respect to $a$, then since the subgroup $A$ is abelian and has index $p^{a}$, it follows that every irreducible character of $P$ has degree at most $|P: A|=p^{a}$. Note also that every abelian $p$-group is suitable with respect to 0 and that every $p$-group having an abelian subgroup of index $p$ is suitable with respect to 1. (To check this last assertion, it suffices to observe that an invariant irreducible character of a normal subgroup of prime index in any finite group is guaranteed to be extendible. This is Corollary 6.20 of [3].) The key to the proof of Theorem A is the following "one-new-degree" theorem. 
(2.3) Theorem. Let $b>a \geq 0$ be integers and suppose that $Q$ is a p-group that is suitable with respect to $a$. Then there exists a p-group $P$ that is suitable with respect to $b$ and such that $\operatorname{cd}(P)=\operatorname{cd}(Q) \cup\left\{p^{b}\right\}$. Furthermore, $P$ can be chosen so that its nilpotence class $c(P)$ is the maximum of 2 and the class $c(Q)$.

Proof. Let $X=Q \times V$, where $V$ is elementary abelian of order $p^{b-a}$ and view $Q$ and $V$ as subgroups of $X$. Since $Q$ is suitable with respect to $a$, we know that $Q$ has an abelian normal subgroup $A$ such that $Q / A$ is elementary abelian of order $p^{a}$, and it follows that $A \triangleleft X$ and that $X / A$ is elementary abelian of order $p^{b}$.

Now let $Y$ be extraspecial of order $p^{2 b+1}$ and let $B$ be a maximal abelian subgroup of $Y$. Then as is well known, $|Y: B|=p^{b}$ and $Y / B$ is elementary abelian, and thus $X / A \cong Y / B$. We can therefore construct the "pullback" $P$, as in Lemma 2.1, and we use the notation of that lemma. As we remarked earlier, $P$ is a $p$-group and $c(P)$ is the maximum of $c(X)=c(Q)$ and $c(Y)=2$, as required.

Now $R S \cong R \times S \cong A \times B$, which is abelian, and $P / R S \cong X / A$, which is elementary abelian of order $p^{b}$. To prove that $P$ is suitable with respect to $b$, therefore, it suffices to show that every linear character of the subgroup $R S$ extends to its stabilizer in $P$. Every such linear character is uniquely of the form $\lambda \mu$, where $R \subseteq \operatorname{ker}(\lambda)$ and $S \subseteq \operatorname{ker}(\mu)$, and because of the uniqueness, we see that the stabilizer of $\lambda \mu$ in $P$ is the intersection of the stabilizers in $P$ of $\lambda$ and $\mu$.

There are just two possibilities for the stabilizer of $\mu$ in $P$. To see why this is so, observe that $\mu$ can be identified with a linear character $\mu_{0}$ of $B \cong R S / S$. (Recall that $B$ is a maximum abelian subgroup of the extraspecial group $Y \cong P / S$.) If $Y^{\prime} \subseteq \operatorname{ker}\left(\mu_{0}\right)$, then $\mu_{0}$ is extendible to $Y$ and hence is invariant in $Y$. Otherwise, $\mu_{0}$ induces irreducibly to $Y$, and hence $B$ is its stabilizer in $Y$. We deduce that either $\mu$ is invariant in (and extends to) $P$ or else the stabilizer of $\mu$ in $P$ is the subgroup $R S$. In the latter case, we see that the stabilizer of $\lambda \mu$ in $P$ is $R S$, and in this situation it is trivially true that $\lambda \mu$ extends to its stabilizer, as required. We can thus suppose that $\mu$ is invariant in $P$ and we let $T$ be the stabilizer of $\lambda$ in $P$. Then $T$ is also the stabilizer of $\lambda \mu$ in $P$ and we must show that $\lambda \mu$ extends to $T$. But since $\mu$ extends to $P$ in this situation, it suffices to show that $\lambda$ extends to $T$.

Now $\lambda$ corresponds to a linear character $\lambda_{0}$ of $A \cong R S / R$. Also, the stabilizer $T_{0}$ of $\lambda_{0}$ in $X=Q \times V$ is the subgroup that corresponds to $T / R$ under the isomorphism $X \cong P / R$. Since $Q$ is suitable, we know that $\lambda_{0}$ extends to its stabilizer in $Q$, and thus it also extends to its stabilizer $T_{0}$ in $X=Q \times V$. It follows from this that $\lambda$ extends to its stabilizer $T$ in $P$, and this completes the proof that $P$ is suitable with respect to $b$, as desired.

Finally, we must show that $\operatorname{cd}(P)=\operatorname{cd}(Q) \cup\left\{p^{b}\right\}$. Since $P / R \cong X=Q \times V$, we see that $Q$ is a homomorphic image of $P$, and thus $\operatorname{cd}(Q) \subseteq \operatorname{cd}(P)$. Similarly, the extraspecial group $Y \cong P / S$ is a homomorphic image of $P$ and we know that $\operatorname{cd}(Y)=\left\{1, p^{b}\right\}$, and thus $p^{b} \in \operatorname{cd}(P)$, as desired. To complete the proof, we must consider an arbitrary character $\chi \in \operatorname{Irr}(P)$ and show that either $\chi(1)=p^{b}$ or $\chi(1) \in \operatorname{cd}(Q)$.

Let $\lambda \mu$ be a linear constituent of $\chi_{R S}$, where, as before, $R \subseteq \operatorname{ker}(\lambda)$ and $S \subseteq$ $\operatorname{ker}(\mu)$. We saw previously that the stabilizer of $\mu$ is either $R S$ or $P$. If the stabilizer of $\mu$ is $R S$, we know that the stabilizer of $\lambda \mu$ is also $R S$, and thus $(\lambda \mu)^{P}$ is irreducible. In this case, $\chi=(\lambda \mu)^{P}$ has degree $|P: R S|=p^{b}$, and there is nothing further to prove. In the remaining case, we know that $\lambda \mu$ extends to its stabilizer $T$ in $P$, and thus since $T / R S$ is abelian, it follows that every irreducible character 
of $T$ that lies over $\lambda \mu$ is linear. We conclude from this that $\chi(1)=|P: T|$. But we know in this case that $T$ corresponds to the stabilizer $T_{0}$ of some linear character $\lambda_{0}$ of $A$ in the group $X=Q \times V$, and we also know that $\lambda_{0}$ extends to $T_{0}$. It follows that the number $|P: T|=\left|X: T_{0}\right|$ lies in the set $\operatorname{cd}(X)=\operatorname{cd}(Q)$. This completes the proof.

Our principal application of Theorem 2.3 is the following, from which Theorem A is immediate.

(2.4) Corollary. Suppose that $Q$ is a p-group that is suitable with respect to $e$, where $p^{e}$ is the largest member of $\operatorname{cd}(Q)$, and let $\mathcal{B}$ be a set of powers of $p$ all of which exceed $p^{e}$. Then there exists a p-group $P$ such that $\operatorname{cd}(P)=\operatorname{cd}(Q) \cup \mathcal{B}$. Furthermore, we can choose $P$ so that $c(P)=c(Q)$ except when $c(Q)=1$ and $\mathcal{B}$ is nonempty, in which case $c(P)=2$.

Proof. We can certainly assume that $\mathcal{B}$ is nonempty and we write $\mathcal{B}=\left\{p^{e_{1}}, p^{e_{2}}, \ldots\right.$, $\left.p^{e_{r}}\right\}$, where $e_{1}<e_{2}<\cdots<e_{r}$. We set $P_{0}=Q$ and we use Theorem 2.3 repeatedly to construct a sequence of $p$-groups $P_{i}$, where $1 \leq i \leq r$. We can do this so that $\operatorname{cd}\left(P_{i}\right)=\operatorname{cd}\left(P_{i-1}\right) \cup\left\{p^{e_{i}}\right\}$ and each of the groups $P_{i}$ is suitable with respect to $e_{i}$. Furthermore, the nilpotence classes of the groups $P_{i}$ will all be equal to $c(Q)$ except when $c(Q)=1$, in which case $c\left(P_{i}\right)=2$ for $i>0$. The group $P=P_{r}$ has the desired properties.

Proof of Theorem A. By [1, we know that there exists a $p$-group $Q$ such that $c(Q)=n$ and $\operatorname{cd}(Q)=\{1, p\}$. Furthermore, $Q$ has an abelian subgroup of index $p$, and thus $Q$ is suitable with respect to 1 . We can now apply Corollary 2.4 with

this group $Q$ and with $\mathcal{B}=\mathcal{S}-\{1, p\}$ to obtain a group $P$ such that $c(P)=n$ and $\operatorname{cd}(P)=\operatorname{cd}(Q) \cup \mathcal{B}=\mathcal{S}$, as desired.

\section{Further APPLICATIONS}

We can also use Corollary 2.4 to give a new proof of the main theorem of [2].

(3.1) Theorem. Let $\mathcal{S}$ be a set of powers of $p$ and assume that $1 \in \mathcal{S}$. Then there exists a p-group $P$ with nilpotence class 2 and $\operatorname{cd}(P)=\mathcal{S}$.

Proof. Let $Q$ be any abelian $p$-group. Then $Q$ is suitable with respect to 0 and $\operatorname{cd}(Q)=\{1\}$. Now apply Corollary 2.4 with $\mathcal{B}=\mathcal{S}-\{1\}$ to obtain the desired group $P$.

If $\mathcal{S}$ is a class-bounding set of powers of $p$, we ask how small the corresponding bound can be. We can use Corollary 2.4 to show that this bound can never be smaller than $p$, and in fact, we have a bit more.

(3.2) Theorem. Let $\mathcal{S}$ be a set of powers of $p$ and assume that $1 \in \mathcal{S}$ and $|\mathcal{S}|>1$. If $n$ is an integer and $2 \leq n \leq p$, then there exists a p-group $P$ with nilpotence class $n$ and $\operatorname{cd}(P)=\mathcal{S}$.

Proof. Let 1 and $p^{e}$ be the two smallest members of $\mathcal{S}$. We claim that there exists a $p$-group $R$ such that $\operatorname{cd}(R)=\left\{1, p^{e}\right\}$ and class $c(R)=p$, and that in addition, $R$ can be chosen so that it has an abelian normal subgroup $B$ such that $R / B$ is elementary abelian of order $p^{e}$. If $e=1$, it is easy to construct such a group: Let $R$ be the wreath product of a cyclic group of order $p$ with itself. If $e>1$, then Example 3.9 of [1] shows that $R$ exists. 
Since $n \leq p$, there is some homomorphic image $Q$ of $R$ with $c(Q)=n$, and we know that $\operatorname{cd}(Q) \subseteq \operatorname{cd}(R)=\left\{1, p^{e}\right\}$. Also, because $n \geq 2$, we see that $Q$ is nonabelian, and thus $\operatorname{cd}(Q)=\left\{1, p^{e}\right\}$. Furthermore, the image $A \subseteq Q$ of $B$ is abelian and $|Q: A| \leq|R: B|=p^{e}$. It follows that $|Q: A|=p^{e}$ and $Q / A \cong R / B$ is elementary abelian.

Now if $\lambda$ is any linear character of $A$, we show that $\lambda$ extends to its stabilizer in $Q$. If some linear character of $Q$ lies over $\lambda$, this is obvious, and otherwise there exists $\chi \in \operatorname{Irr}(Q)$ lying over $\lambda$ with $\chi(1)=p^{e}$. Since $|P: A|=p^{e}$, it follows that $\lambda^{G}=\chi$, and thus the full stabilizer of $\lambda$ in $P$ is the subgroup $A$. In this case too, we see that $\lambda$ extends to its stabilizer, and thus $Q$ is suitable with respect to $e$.

We can now apply Corollary 2.4 with $\mathcal{B}=\mathcal{S}-\left\{1, p^{e}\right\}$ to obtain a group $P$ with the desired properties.

\section{Some Questions}

The big remaining question, of course, is the following.

Question 1. If $\mathcal{S}$ is a set of powers of $p$ that contains 1 but not $p$, must $\mathcal{S}$ be class bounding?

It seems hard to find sets that one can prove to be class bounding, and so the answer to Question 1 may well be "no". In fact, the assumption that $\mathcal{S}$ is class bounding may turn out to be extremely restrictive. It may be, for example, that if $\mathcal{S}$ is class bounding and $\operatorname{cd}(P)=\mathcal{S}$, then the structure of $P$ is very tightly constrained. For example, we do not know the answer to the following.

Question 2. Does there exist a $p$-group $P$ such that $\operatorname{cd}(P)$ is class bounding and $P$ is not metabelian?

If the answer to Question 2 is "no", then the answer to Question 1 must also be "no". This is because there exist $p$-groups $P$ of arbitrarily large derived length for which $p \notin \operatorname{cd}(P)$. For example, we can take $P$ to be a Sylow $p$-subgroup of $G L\left(n, p^{e}\right)$ where $e>1$ and $n$ is large. In this situation, it is known (see [4]) that $\operatorname{cd}(P)$ consists of powers of $p^{e}$, and so does not contain $p$, but the nilpotence class and derived length of $P$ are unboundedly large as $n$ grows.

For every class-bounding set $\mathcal{S}$, there is some integer $b(\mathcal{S})$, which is the largest possible nilpotence class for a $p$-group $P$ such that $\operatorname{cd}(P)=\mathcal{S}$. By Theorem 3.2, we know that $b(\mathcal{S}) \geq p$ for every class-bounding set other than $\{1\}$, but is there an upper bound for $b(\mathcal{S})$ ?

Question 3. Can $b(\mathcal{S})$ be arbitrarily large for a class-bounding set $\mathcal{S}$ ?

If the answer to Question 3 is "no", then again, by considering a Sylow $p$ subgroup of $G L\left(n, p^{e}\right)$ with $e>1$ and $n$ large, we see that the answer to Question 1 must be "no".

We know of just one class-bounding set $\mathcal{S}$ for which we can show that $b(\mathcal{S})$ exceeds $p$ : the set $\mathcal{S}=\{1,4,16\}$. (This set is class bounding because it has cardinality 3 and does not contain $p=2$.) If $P$ is a Sylow 2-subgroup of $G L(4,4)$, then it is well known that $P$ has class 3 . Also, all members of $\operatorname{cd}(P)$ are powers of 4 and it is easy to see that $P$ has an abelian subgroup of index 16. It follows that $\operatorname{cd}(P) \subseteq\{1,4,16\}$, and it is not too hard to show that all three of these degrees occur, and thus $\operatorname{cd}(P)=\{1,4,16\}$. This suggests what is probably the least ambitious of our questions. 
Question 4. If $p>2$, does there exist a $p$-group $P$ of class $p+1$ such that $\operatorname{cd}(P)=$ $\left\{1, p^{2}, p^{4}\right\}$ ?

\section{REFERENCES}

[1] I. M. Isaacs and D. S. Passman, A characterization of groups in terms of the degrees of their characters. II. Pacific J. Math. 24 (1968) 467-510. MR 39:7001

[2] I. M. Isaacs, Sets of $p$-powers as irreducible character degrees. Proc. Amer. Math. Soc. 96 (1986) 551-552. MR 87d:20013

[3] I. M. Isaacs, Character Theory of Finite Groups, Dover, New York, 1994. MR 57:417

[4] I. M. Isaacs, Characters of groups associated with finite algebras. J. Algebra 177 (1995) 708730. MR 96k:20011

[5] I. M. Isaacs and A. Moretó, Character degrees and the nilpotence class of a $p$-group, J. of Algebra 238 (2001) 827-842. CMP 2001:11

[6] M. C. Slattery, Character degrees and nilpotence class in p-groups, J. of Austral. Math. Soc. (Series A) 57 (1994) 76-80. MR 95d:20013

Department of Mathematics, University of Wisconsin, 480 Lincoln Drive, Madison, WISCONSIN 53706

E-mail address: isaacs@math.wisc.edu

Department of Mathematics, Statistics and Computer Science, Marquette UniverSity, P.O. Box 1881, Milwaukee, Wisconsin 53201

E-mail address: mikes@mscs.mu.edu 